\title{
Questionnaire 2004: une formation post- graduée meilleure encore, en quinze minutes
}

Max Giger, président de la Commission pour la formation postgraduée et continue FMH, Winterthour

L'enquête 2003 a été réalisée avec un nouveau questionnaire. Dans de nombreux établissements de formation postgraduée, les résultats ont provoqué un débat bienvenu sur l'efficacité de la formation postgraduée. La raison d'être et l'organisation de l'année à option doivent être revues. Outre les questions générales, le questionnaire 2004 remanié porte également sur la mise en évidence des erreurs et la médecine basée sur des données probantes.

Le questionnaire sur l'appréciation de la formation postgraduée par les médecins-assistants est envoyé ces jours-ci aux établissements de formation postgraduée. Cette enquête menée pour la huitième fois a pour but, à l'instar des précédentes, de connaître l'avis des médecins en formation postgraduée. Nous espérons que les personnes concernées participeront en très grand nombre à cette enquête, conçue précisément en faveur de tous ceux qui se forment en vue d'un titre de spécialiste. Cette enquête est l'une des trois méthodes permettant de garantir la qualité de la formation postgraduée. Le temps nécessaire pour répondre au questionnaire dure un petit quart d'heure.

L'enquête de cette année porte sur les deux thèmes traités durant l'année «Culture de l'erreur (mise en évidence des erreurs)» et «Application de la médecine basée sur des données probantes (médecine factuelle)». Nous espérons pouvoir vous informer des premiers résultats à la fin de l'automne.

Cette année également, les médecins en formation postgraduée et les formateurs sont invités à s'exprimer de façon critique. J'accepte volontiers de recevoir personnellement des critiques mais elles peuvent aussi être communiquées publiquement sur le site internet de la FPPC (www.fmh.ch/awf). Les critiques constructives nous permettent en effet d'améliorer la qualité du questionnaire d'une année à l'autre et de faire le point sur la situation des établissements de formation postgraduée. 